\title{
An analysis of underweight status on 30 - day outcomes after breast reconstruction
}

\author{
Anas Eid ${ }^{1}$, Jon P. Ver Halen ${ }^{1,2}$ \\ ${ }^{1}$ Division of Plastic, Reconstructive, and Hand Surgery, Baptist Cancer Center, Germantown, TN 38139, USA. \\ ${ }^{2}$ Department of Surgery, Vanderbilt-Ingram Cancer Center, Nashville, TN 37232, USA.
}

Address for correspondence: Dr. Jon P. Ver Halen, Division of Plastic, Reconstructive, and Hand Surgery, Baptist Cancer Center, Germantown, TN 38139, USA. Email: jpverhalen@gmail.com

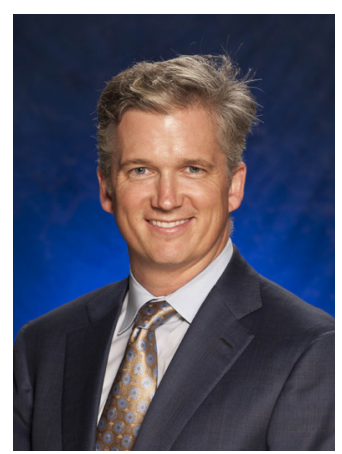

Dr. Jon P. Ver Halen is currently an Associate Professor with the Texas A\&M School of Medicine, Department of Surgery. He is also Associate Program Director of the Plastic Surgery Residency, and Program Director of the Microvascular Surgery Fellowship.

\begin{abstract}
\end{abstract}
Aim: To examine the impact of underweight body mass index (BMI) values on breast reconstruction outcomes. Methods: The American College of Surgeons National Surgical Quality Improvement Program (ACS-NSQIP) database was retrospectively reviewed for all patients who underwent breast reconstruction between 2006 and 2011. Patients were first stratified by breast reconstruction modality into prosthetic or autologous cohorts, and second by BMI values into underweight (BMI < 18.5), normal to overweight (reference, BMI 18.5-29.99), moderate obesity (BMI 30-34.99), severe obesity (BMI 35-39.99), and morbid obesity cohorts. Multivariate logistic regression models were used to determine independent predictors of complications. Results: With regard to prosthetic breast reconstruction patients, obese patients demonstrated increased rates of surgical complications, while underweight patients did not have any differences on multivariable analysis. With respect to autologous reconstruction, risk-adjusted multivariate regression models showed a dose dependent response between obesity and risk for surgical complications and reoperation, but not for underweight patients. Conclusion: On multivariable analysis of over 4,600 patients, there were no significant differences in the rates of adverse events between underweight patients $(\mathrm{BMI}<18.5)$ and their reference-weight counterparts, in spite of a significant increase in surgical and medical complication rates in underweight patients on univariate analysis.

Key words:

Breast reconstruction; underweight; complications; body mass index; obesity

\begin{tabular}{|l|l|}
\hline \multicolumn{2}{|c|}{ Access this article online } \\
\hline Quick Response Code: & Website: \\
\hline & Www.parjournal.net \\
\cline { 2 - 3 } & DOI: \\
\hline
\end{tabular}

This is an open access article distributed under the terms of the Creative Commons Attribution-NonCommercial-ShareAlike 3.0 License, which allows others to remix tweak and build upon the work non-commercially, as long as the author is credited and the new creations are licensed under the identical terms.

For reprints contact: service@oaepublish.com

How to cite this article: Eid A, Ver Halen JP. An analysis of underweight status on 30-day outcomes after breast reconstruction. Plast Aesthet Res 2016;3:8-14.

Received: 05-05-2015; Accepted: 18-06-2015 


\section{INTRODUCTION}

Much has been published regarding the risks of obesity on medical outcomes. The prevalence of obesity among adults in the United States has been steadily increasing over the past several decades such that today over 1 in every 3 adults is obese [body mass index (BMI) $>30 \mathrm{~kg} / \mathrm{m}^{2}$ ], and nearly 1 in every 10 adults is morbidly obese $\left(\mathrm{BMI}>40 \mathrm{~kg} / \mathrm{m}^{2}\right) \cdot{ }^{[1,2]}$ Obesity is a multi-system disease process which confers increased risk of medical comorbidities including hypertension (HTN), coronary artery disease, and diabetes mellitus (DM), and increases the risk of surgical morbidity. ${ }^{[3,4]}$ Similarly, extremes of underweight have recently been described as a risk factor for surgery. ${ }^{[5-7]}$ Several recent studies of critically and chronically ill patients, ${ }^{[8-11]}$ and of patients undergoing certain procedures ${ }^{[12-15]}$ suggest that overweight and obese patients may paradoxically have better outcomes than underweight patients, given an increased risk for death and catastrophic complications in the latter patients.

As many as $40 \%$ of women undergoing mastectomies in the USA, they are now seeking post-mastectomy breast reconstruction. ${ }^{[16-19]}$ While much recent literature has detailed an association between obesity and poor surgical outcomes, ${ }^{[20-23]}$ other studies have failed to demonstrate an increased risk of death or severe complications in these patients. ${ }^{[24-26]}$ Conversely, very little has been written about the risk of underweight patients undergoing breast reconstruction. Such studies have been compromised by small sample sizes, single-institutional bias, retrospective study design, limited patient follow-up, inconsistent definitions of underweight, types of surgical procedures included, and outcomes studied. ${ }^{[27-30]}$

In an effort to better understand the influence of BMI on outcomes following breast reconstruction, we examined the National Surgical Quality Improvement Program (NSQIP) datasets. We aim to define and benchmark the risks and outcomes associated with breast reconstruction in underweight patients. We hypothesized that patients who are at extremes of low BMI would have a higher risk of adverse outcomes.

\section{METHODS}

\section{Patient population}

All patients with "Plastics" recorded as their primary surgical team were isolated from the 2006-2011 NSQIP database. Patients were stratified into either "prosthetic" or "autologous" reconstruction cohorts, based on ACSNSQIP classification. ACS-NSQIP tracks procedures based on Current Procedural Terminology (CPT) codes. Specific CPT codes used for each cohort include: 19340 (immediate breast reconstruction with implant), 19342 (delayed breast reconstruction with implant), 19357 (breast reconstruction with tissue expander), 19361 (breast reconstruction with latissimus dorsi flap), 19364 (breast reconstruction with free flap), 19367 [breast reconstruction transverse rectus abdominis musculocutaneous (TRAM) flap] and 19368 (breast reconstruction with TRAM flap, with microvascular anastomosis). Patients undergoing multiple types of reconstruction (e.g. latissimus dorsi flap + implant, or different types of reconstruction on each side) were excluded from analysis. Similarly, only patients with total breast reconstruction using the above-mentioned codes were included. Thus, patients undergoing breast reconstruction via fat grafting (CPT code 15770) or local flap closure (14301, 14302, 15734) were excluded from analysis. Breast reconstruction patients were further categorized into prosthetic and autologous reconstruction cohorts. Similar preoperative demographic and postoperative outcomes analyses were carried out separately in the prosthetic and autologous populations groups. Multivariate regression analysis was also conducted in similar fashion to the overall population.

Table 1: Prosthetic breast reconstruction patient clinical characteristics, stratified by body mass index, $n$ (\%)

\begin{tabular}{|c|c|c|c|c|c|}
\hline & $\begin{array}{c}\text { Underweight } \\
(<18.5, n=116)\end{array}$ & $\begin{array}{l}\text { Normal to overweight } \\
(18.5-29.99, n=2,543)\end{array}$ & $\begin{array}{l}\text { Moderate obesity } \\
(30-34.99, n=511)\end{array}$ & $\begin{array}{c}\text { Severe obesity } \\
(35-39.99, n=229)\end{array}$ & $\begin{array}{l}\text { Morbid obesity } \\
(\geq 40, n=114)\end{array}$ \\
\hline Age & $48.12 \pm 12.04$ & $51.43 \pm 11.55$ & $53.988 \pm 10.58$ & $54.60 \pm 10.92$ & $52.54 \pm 10.56$ \\
\hline Hypertension & $9(7.76)$ & $474(18.64)$ & $204(39.92)$ & $125(54.59)$ & $56(49.12)$ \\
\hline Diabetes & $2(1.72)$ & $71(2.79)$ & $57(11.15)$ & $37(16.16)$ & $18(15.79)$ \\
\hline COPD & $2(1.72)$ & $16(0.63)$ & $4(0.78)$ & $7(3.06)$ & $2(1.75)$ \\
\hline Dyspnea & $3(2.59)$ & $58(2.43)$ & $17(3.33)$ & $17(7.42)$ & $10(8.77)$ \\
\hline History of TIA or CVA & $0(0.00)$ & $14(0.59)$ & $8(1.57)$ & $2(0.87)$ & $3(2.63)$ \\
\hline Prior $\mathrm{PCl}$ or $\mathrm{PCS}$ & $0(0.00)$ & $21(0.83)$ & $8(1.57)$ & $4(1.75)$ & $0(0.00)$ \\
\hline Active smoking & $20(17.24)$ & $344(13.53)$ & $62(12.13)$ & $25(10.92)$ & $16(14.04)$ \\
\hline Alcohol use & $3(2.59)$ & $28(1.10)$ & $4(0.78)$ & $2(0.87)$ & $1(0.88)$ \\
\hline Chronic steroid use & $0(0.00)$ & $22(0.87)$ & $2(0.39)$ & $3(1.31)$ & $4(3.51)$ \\
\hline Chemotherapy within 30 days & $3(2.59)$ & $79(3.11)$ & $16(3.13)$ & $5(2.18)$ & $4(3.51)$ \\
\hline Radiation within 90 days & $0(0.00)$ & $12(0.47)$ & $3(0.59)$ & $0(0.00)$ & $1(0.88)$ \\
\hline Wound infection within 30 days & $1(0.86)$ & $36(1.42)$ & $6(1.17)$ & $1(0.44)$ & $0(0.00)$ \\
\hline Prior operation within 30 days & $2(1.72)$ & $20(0.79)$ & $6(1.17)$ & $2(0.87)$ & $0(0.00)$ \\
\hline Outpatient cases & $81(69.82)$ & $1,781(70.03)$ & $380(74.36)$ & $162(70.74)$ & $73(64.04)$ \\
\hline Emergent cases & $1(0.86)$ & $14(0.55)$ & $3(0.59)$ & $1(0.05)$ & $1(0.88)$ \\
\hline Sum of relative value units & $34.30 \pm 17.90$ & $33.69 \pm 19.71$ & $34.15 \pm 20.13$ & $33.59 \pm 18.06$ & $36.46 \pm 23.04$ \\
\hline Operative time $(\mathrm{h})$ & $2.27 \pm 2.18$ & $2.17 \pm 1.32$ & $2.22 \pm 1.43$ & $2.20 \pm 1.18$ & $2.46 \pm 1.78$ \\
\hline
\end{tabular}

COPD: chronic obstructive pulmonary disease; TIA: transient ischemic attack; CVA: cerebrovascular accident; PCI: previous coronary intervention; PCS: previous cardiac surgery 
Table 2: Autologous breast reconstruction patient clinical characteristics, stratified by body mass index, $\boldsymbol{n}(\%)$

\begin{tabular}{|c|c|c|c|c|c|}
\hline & $\begin{array}{c}\text { Underweight } \\
(<18.5, n=20)\end{array}$ & $\begin{array}{c}\text { Normal to overweight } \\
(18.5-29.99, n=706)\end{array}$ & $\begin{array}{c}\text { Moderate obesity } \\
(30-34.99, n=281)\end{array}$ & $\begin{array}{c}\text { Severe obesity } \\
(35-39.99, n=109)\end{array}$ & $\begin{array}{l}\text { Morbid obesity } \\
(\geq 40, n=47)\end{array}$ \\
\hline Age & $48.42 \pm 11.71$ & $51.35 \pm 10.05$ & $52.08 \pm 8.90$ & $51.66 \pm 9.15$ & $50.81 \pm 9.56$ \\
\hline Hypertension & $4(20.00)$ & $165(25.31)$ & $95(33.81)$ & $48(44.04)$ & $21(44.68)$ \\
\hline Diabetes & $0(0.00)$ & $22(3.37)$ & $17(6.05)$ & $16(14.68)$ & $6(12.77)$ \\
\hline COPD & $0(0.00)$ & $5(0.77)$ & $1(0.36)$ & $0(0.00)$ & $0(0.00)$ \\
\hline Dyspnea & $1(5.00)$ & $15(2.30)$ & $7(2.49)$ & $4(3.67)$ & $3(6.38)$ \\
\hline History of TIA or CVA & $0(0.00)$ & $7(1.07)$ & $1(0.36)$ & $1(0.92)$ & $1(2.13)$ \\
\hline Prior $\mathrm{PCl}$ or $\mathrm{PCS}$ & $0(0.00)$ & $4(0.61)$ & $1(0.36)$ & $0(0.00)$ & $0(0.00)$ \\
\hline Active smoking & $3(15.00)$ & $75(11.50)$ & $28(9.96)$ & $7(6.42)$ & $9(19.15)$ \\
\hline Alcohol use & $0(0.00)$ & $6(0.92)$ & $2(0.71)$ & $0(0.00)$ & $1(2.13)$ \\
\hline Chronic steroid use & $1(5.00)$ & $7(1.07)$ & $2(0.71)$ & $1(0.92)$ & $0(0.00)$ \\
\hline Chemotherapy within 30 days & $1(5.00)$ & $29(4.44)$ & $14(4.98)$ & $3(2.75)$ & $0(0.00)$ \\
\hline Radiation within 90 days & $0(0.00)$ & $6(0.92)$ & $2(0.71)$ & $2(1.83)$ & $0(0.00)$ \\
\hline Wound infection within 30 days & $1(5.00)$ & $21(3.22)$ & $5(1.78)$ & $4(3.67)$ & $1(2.13)$ \\
\hline Prior operation within 30 days & $0(0.00)$ & $22(3.37)$ & $7(2.49)$ & $3(2.75)$ & $2(4.26)$ \\
\hline Outpatient cases & $2(10.00)$ & $75(11.50)$ & $22(7.83)$ & $5(4.59)$ & $7(14.89)$ \\
\hline Emergent cases & $1(5.00)$ & $3(0.46)$ & $1(0.36)$ & $0(0.00)$ & $0(0.00)$ \\
\hline Sum of relative value units & $48.02 \pm 31.37$ & $47.28 \pm 24.73$ & 48. $79 \pm 24.39$ & $48.46 \pm 26.30$ & $47.14 \pm 28.81$ \\
\hline Operative time $(\mathrm{h})$ & $5.82 \pm 2.42$ & $6.09 \pm 3.12$ & $6.61 \pm 3.41$ & $6.38 \pm 3.64$ & $6.05 \pm 3.63$ \\
\hline
\end{tabular}

TIA: transient ischemic attack; COPD: chronic obstructive pulmonary disease; CVA: cerebrovascular accident; PCl: previous coronary intervention; PCS: previous cardiac surgery

\section{Outcomes}

Our primary outcomes of interest were: 30-day surgical complications, medical complications, reoperation, and mortality. Surgical complication was defined as having $\geq 1$ of the following ACS-NSQIP post-operative adverse events: superficial surgical site infection (SSI), deep surgical site infection, organ/space surgical site infection, wound disruption/dehiscence, or graft/prosthesis failure. Medical complications included: pneumonia, unplanned intubation, pulmonary embolism (PE), failure to wean from ventilator, renal insufficiency, progressive renal failure, urinary tract infection, stroke, coma, peripheral neurologic deficiency, cardiac arrest, myocardial infarction, bleeding requiring a transfusion, deep venous thrombosis (DVT), and sepsis/septic shock.

\section{Statistical analysis}

Patients were stratified into BMI categories as follows: underweight, BMI < 18.5; normal to overweight, BMI 18.529.99; moderately obese, BMI 30-34.99; severely obese, BMI 35-39.99; and morbidly obese, BMI $\geq 40$. Patient demographics and clinical characteristics were tracked as potential cofounders. Chi-square analysis was used to compare categorical variables and one-way ANOVA tests were used to analyze continuous variables.

Multivariable logistic regression analysis was utilized to investigate the impact of BMI values on outcomes. Preoperative variables with $\geq 10$ occurrences and $P \leq 20$ on bivariate screening were included in the analysis. All analysis was conducted using Statistical Product and Service Solutions (SPSS) version 21 (Chicago, IL). P values less than 0.05 were statistically significant. For statistical evaluation, the reference population was defined as the normal-weight cohort (i.e. BMI 18.5-29.99).

\section{RESULTS}

In review of the 25,346 plastic surgery patients extracted from the database, 4,676 patients met criteria for study inclusion. Three-fourths $(3,513)$ of the reconstruction patients received prosthetic reconstruction and the remaining quarter $(1,163)$ underwent autologous tissue based reconstruction. Rates of hypertension, diabetes, and dyspnea increased as BMI values increased in both prosthetic and autologous cohorts [Tables 1 and 2]. For statistical evaluation, the reference population was defined as the normal-weight cohort (i.e. BMI 18.5-29.99).

On univariate analysis, in the prosthetic patient population, adverse events (AE) increased from underweight, to reference, to obese patients [Table 3, Figure 1]. Total complications rose from $1.7 \%, 3.3 \%$, to $11.4 \%$ in underweight, reference, and morbidly obese patients, respectively $(P<$ 0.001 ). Similarly, surgical complications increased from $1.7 \%$, $2.9 \%$, to $11.4 \%$ as weight strata increased $(P<0.001)$. Medical complications were significantly increased in underweight and obese patients, compared to reference weight patients increased $(1.7 \%$ for underweight, $2.4 \%$ for obese, and $0.8 \%$ for reference weight patients) $(P=0.009)$. Finally, reoperation rates increased as weight strata increased $(0 \%, 3.6 \%$, to $8.8 \%$, respectively) $(P=0.001)$. There were no deaths in the prosthetic breast reconstruction cohort.

With respect to autologous reconstruction, complication rates increased when patients were at extremes of weight, whether underweight or overweight [Figure 2]. While the reference population (i.e. BMI 20-30) had a rate of total complications of $16.6 \%$, underweight patients had a total rate of $20 \%$, and overweight patients' complication rate increased to $40.43 \%(P<0.001)$. Similarly, surgical complications increased from $6.9 \%$ to $15 \%$ and $29.79 \%$ in underweight and obese patients, respectively $(P<0.001)$. 
Table 3: Postoperative complications following prosthetic breast reconstruction, stratified by body mass index, univariate analysis, $n(\%)$

\begin{tabular}{|c|c|c|c|c|c|c|}
\hline & $\begin{array}{c}\text { Underweight } \\
(<18.5, n=116)\end{array}$ & $\begin{array}{l}\text { Normal to overweight } \\
(18.5-29.99, n=2,543)\end{array}$ & $\begin{array}{c}\text { Moderate obesity } \\
(30-34.99, n=511)\end{array}$ & $\begin{array}{c}\text { Severe obesity } \\
(35-39.99, n=229)\end{array}$ & $\begin{array}{l}\text { Morbid obesity } \\
(\geq 40, n=114)\end{array}$ & $P$ \\
\hline Total complications & $2(1.72)$ & $85(3.34)$ & $28(5.48)$ & $19(8.30)$ & $13(11.40)$ & $<0.001^{*}$ \\
\hline Surgical complications & $2(1.72)$ & $73(2.87)$ & $21(4.11)$ & $15(6.55)$ & $13(11.40)$ & $<0.001^{*}$ \\
\hline Wound infection & $2(1.72)$ & $57(2.24)$ & $18(3.52)$ & $15(6.55)$ & $10(8.77)$ & $<0.001^{*}$ \\
\hline Superficial SSI & $0(0.00)$ & $31(1.22)$ & $12(2.35)$ & $4(1.75)$ & $2(1.75)$ & 0.272 \\
\hline Deep SSI & $0(0.00)$ & $16(0.63)$ & $3(0.59)$ & $6(2.62)$ & $2(1.75)$ & $0.006^{*}$ \\
\hline Organ/space SSI & $2(1.30)$ & $12(0.47)$ & $3(0.59)$ & $5(2.18)$ & $6(5.26)$ & $<0.001^{*}$ \\
\hline Dehiscence & $0(0.00)$ & $19(0.75)$ & $3(0.59)$ & $2(0.87)$ & $4(3.51)$ & $0.02^{*}$ \\
\hline Prosthesis failure & $0(0.00)$ & $4(0.16)$ & $2(0.39)$ & $1(0.44)$ & $1(0.88)$ & 0.384 \\
\hline Medical complications & $2(1.72)$ & $20(0.79)$ & $12(2.35)$ & $5(2.18)$ & $1(0.88)$ & $0.009^{*}$ \\
\hline Pneumonia & $0(0.00)$ & $1(0.04)$ & $0(0.00)$ & $1(0.44)$ & $0(0.00)$ & 0.17 \\
\hline Reintubation & $0(0.00)$ & $1(0.04)$ & $0(0.00)$ & $0(0.00)$ & $0(0.00)$ & 0.976 \\
\hline $\mathrm{PE}$ & $0(0.00)$ & $2(0.08)$ & $2(0.39)$ & $1(0.44)$ & $0(0.00)$ & 0.313 \\
\hline Ventilator $>48 \mathrm{~h}$ & $0(0.00)$ & $0(0.00)$ & $1(0.20)$ & $0(0.00)$ & $0(0.00)$ & 0.209 \\
\hline Renal insufficiency & $0(0.00)$ & $1(0.04)$ & $0(0.00)$ & $0(0.00)$ & $0(0.00)$ & 0.976 \\
\hline Acute renal failure & $0(0.00)$ & $0(0.00)$ & $1(0.20)$ & $0(0.00)$ & $0(0.00)$ & 0.209 \\
\hline UTI & $0(0.00)$ & $4(0.16)$ & $3(0.59)$ & $1(0.44)$ & $0(0.00)$ & 0.329 \\
\hline Stroke & $0(0.00)$ & $1(0.04)$ & $0(0.00)$ & $0(0.00)$ & $0(0.00)$ & 0.976 \\
\hline Coma & $0(0.00)$ & $0(0.00)$ & $0(0.00)$ & $0(0.00)$ & $0(0.00)$ & - \\
\hline Peripheral neuro deficiency & $0(0.00)$ & $0(0.00)$ & $0(0.00)$ & $0(0.00)$ & $0(0.00)$ & - \\
\hline Cardiac arrest & $0(0.00)$ & $0(0.00)$ & $0(0.00)$ & $0(0.00)$ & $0(0.00)$ & - \\
\hline Myocardial Infarction & $0(0.00)$ & $0(0.00)$ & $0(0.00)$ & $0(0.00)$ & $0(0.00)$ & - \\
\hline Bleed requiring transfusion & $0(0.00)$ & $4(0.16)$ & $5(0.98)$ & $0(0.00)$ & $0(0.00)$ & $0.013^{*}$ \\
\hline DVT & $0(0.00)$ & $0(0.00)$ & $0(0.00)$ & $1(0.44)$ & $0(0.00)$ & $0.006^{*}$ \\
\hline Sepsis/septic shock & $1(0.86)$ & $6(0.24)$ & $3(0.59)$ & $1(0.44)$ & $1(0.88)$ & 0.646 \\
\hline Reoperation & $0(0.00)$ & $91(3.58)$ & $20(3.91)$ & $18(7.86)$ & $10(8.77)$ & $0.001^{*}$ \\
\hline Death & $0(0.00)$ & $0(0.00)$ & $1(0.20)$ & $0(0.00)$ & $0(0.00)$ & 0.209 \\
\hline
\end{tabular}

*Denotes significant value, $P<0.05$. SSI: superficial surgical site infection; PE: pulmonary embolism; DVT: deep venous thrombosis; UTI: urinary tract infection

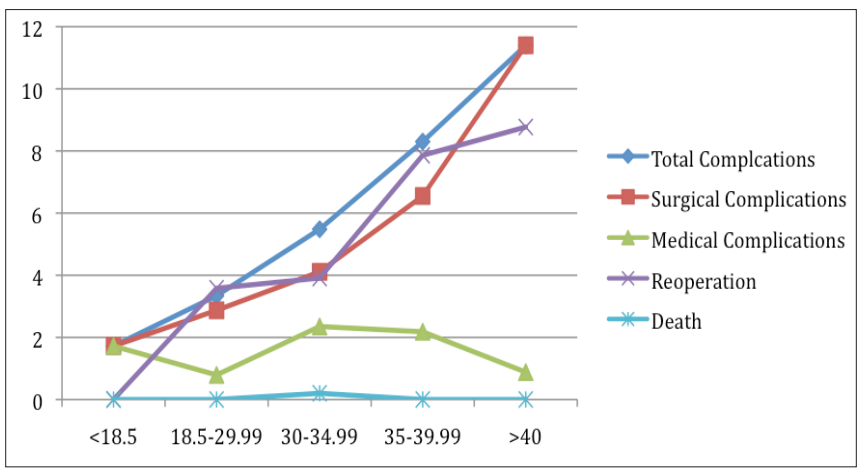

Figure 1: Incidence of adverse events vs. body mass index range, for prosthetic breast reconstruction cohort

In contrast, underweight patients had the lowest rate of medical complications $(5 \%)$, compared to the reference population $(11.2 \%)$, or obese patients $(23.4 \%)(P=0.005)$ [Table 4]. Reoperation rates also increased from 5\%, $9 \%$, to $29.79 \%$ in the underweight, reference, and obese populations, respectively $(P<0.001)$. There was one death in the autologous reconstruction cohort, in the reference weight subgroup (data not significant).

Multivariate regression analysis demonstrated a different picture. With respect to prosthetic reconstruction, only severely and morbidly obese patients had an elevated odds of having a surgical complication [Table 5]; the severely obese were also at risk for reoperation. Additionally, patients with moderate obesity had a $28.9 \%$ increase in their risk for incurring a medical complication. Interestingly, underweight patients appeared to have decreased risk of complications or reoperation, although these numbers did not reach significance.

On multivariate analysis, a strong connection between BMI and autologous reconstruction outcomes was present. Specifically, there was a significant incremental increase in odds for surgical complications when transitioning from reference weight to morbid obesity (ranging from 1.35 to 3.31) [Table 6]. Individuals with a BMI over 35 also had significant risk for reoperation. Medical complications rose as BMI increased, although data did not reach significance. Similarly, underweight patients had an elevated risk of surgical complications; however this data did not reach significance $(P=0.062)$.

\section{DISCUSSION}

This study defines and benchmarks risks and outcomes at 30 days associated with breast reconstruction, utilizing a detailed stratification method, including a categorization of underweight patients. We found 4,676 patients who underwent breast reconstruction during this period, of whom $3,513(75.1 \%)$ underwent prosthetic reconstruction, and 1,163 (24.9\%) underwent autologous reconstruction. Of the total 4,676 patients, $136(3 \%)$ were BMI < 18.5, 3,249 (69.5\%) were BMI 18.5-30, 792 (16.9\%) were BMI 30-34.99, 338 (7.2\%) were BMI 35-39.99, and 161 (3.4\%) were BMI > 40 .

We found significant differences in the groups, with regard to preoperative variables. With regards to prosthetic reconstruction, underweight patients tended to be younger, 
Table 4: Postoperative complications following autologous breast reconstruction, stratified by body mass index, univariate analysis, $n(\%)$

\begin{tabular}{|c|c|c|c|c|c|c|}
\hline & $\begin{array}{c}\text { Underweight } \\
(<18.5, n=20)\end{array}$ & $\begin{array}{l}\text { Normal to overweight } \\
(18.5-29.99, n=706)\end{array}$ & $\begin{array}{c}\text { Moderate obesity } \\
(30-34.99, n=281)\end{array}$ & $\begin{array}{c}\text { Severe obesity } \\
(35-39.99, n=109)\end{array}$ & $\begin{array}{c}\text { Morbid obesity } \\
(\geq 40, n=47)\end{array}$ & $P$ \\
\hline Total complications & $4(20.00)$ & $117(16.57)$ & $64(22.78)$ & $40(36.70)$ & $19(40.43)$ & $<0.001^{*}$ \\
\hline Surgical complications & $3(15.00)$ & $50(7.08)$ & $33(11.74)$ & $21(19.27)$ & $14(29.79)$ & $<0.001^{*}$ \\
\hline Wound infection & $1(5.00)$ & $35(4.61)$ & $24(8.54)$ & $15(13.76)$ & $12(25.53)$ & $<0.001^{*}$ \\
\hline Superficial SSI & $1(5.00)$ & $19(2.69)$ & $19(6.76)$ & $10(9.17)$ & $7(14.89)$ & $<0.001^{*}$ \\
\hline Deep SSI & $1(5.00)$ & $14(1.98)$ & $5(1.78)$ & $2(1.83)$ & $3(6.38)$ & 0.353 \\
\hline Organ/space SSI & $0(0.00)$ & $2(0.28)$ & $1(0.36)$ & $4(3.67)$ & $2(4.26)$ & $<0.001^{*}$ \\
\hline Dehiscence & $1(5.00)$ & $6(0.85)$ & $3(1.07)$ & $3(2.75)$ & $0(0.00)$ & 0.324 \\
\hline Flap failure & $1(5.00)$ & $13(1.84)$ & $11(3.91)$ & $7(6.42)$ & $2(4.26)$ & $0.032^{*}$ \\
\hline Medical complications & $1(5.00)$ & 79 (11.19) & $43(15.30)$ & $23(21.10)$ & $11(23.40)$ & $0.005^{*}$ \\
\hline Pneumonia & $0(0.00)$ & $2(0.28)$ & $0(0.00)$ & $1(0.92)$ & $0(0.00)$ & 0.592 \\
\hline Reintubation & $0(0.00)$ & $4(0.57)$ & $1(0.36)$ & $2(1.83)$ & $0(0.00)$ & 0.471 \\
\hline PE & $0(0.00)$ & $0(0.00)$ & $4(1.42)$ & $1(0.92)$ & $0(0.00)$ & $0.043^{*}$ \\
\hline Ventilator $>48 \mathrm{~h}$ & $0(0.00)$ & $2(0.28)$ & $2(0.71)$ & $1(0.92)$ & $0(0.00)$ & 0.773 \\
\hline Renal insufficiency & $0(0.00)$ & $0(0.00)$ & $0(0.00)$ & $0(0.00)$ & $0(0.00)$ & - \\
\hline Acute renal failure & $0(0.00)$ & $0(0.00)$ & $0(0.00)$ & $0(0.00)$ & $1(2.13)$ & $<0.001^{*}$ \\
\hline UTI & $0(0.00)$ & $6(0.85)$ & $2(0.71)$ & $0(0.00)$ & $1(2.13)$ & 0.677 \\
\hline Stroke & $0(0.00)$ & $0(0.00)$ & $0(0.00)$ & $0(0.00)$ & $0(0.00)$ & - \\
\hline Coma & $0(0.00)$ & $0(0.00)$ & $0(0.00)$ & $0(0.00)$ & $0(0.00)$ & - \\
\hline Peripheral neuro deficiency & $0(0.00)$ & $0(0.00)$ & $0(0.00)$ & $0(0.00)$ & $0(0.00)$ & - \\
\hline Cardiac arrest & $0(0.00)$ & $1(0.14)$ & $0(0.00)$ & $0(0.00)$ & $0(0.00)$ & 0.954 \\
\hline Myocardial infarction & $0(0.00)$ & $1(0.14)$ & $1(0.36)$ & $9(8.26)$ & $9(19.15)$ & 0.923 \\
\hline Bleed requiring transfusion & $1(5.00)$ & $63(8.92)$ & $28(9.96)$ & $17(15.60)$ & $6(12.77)$ & 0.226 \\
\hline DVT & $0(0.00)$ & $4(0.57)$ & $5(1.78)$ & $1(0.92)$ & $0(0.00)$ & 0.388 \\
\hline Sepsis/septic shock & $0(0.00)$ & $6(0.85)$ & $7(2.49)$ & $4(3.67)$ & $3(6.38)$ & $0.011^{*}$ \\
\hline Reoperation & $1(5.00)$ & $63(8.92)$ & $35(12.46)$ & $21(19.27)$ & $14(29.79)$ & $<0.001^{*}$ \\
\hline Death & $0(0.00)$ & $1(0.14)$ & $0(0.00)$ & $0(0.00)$ & $0(0.00)$ & 0.954 \\
\hline
\end{tabular}

*Denotes significant value, $P<0.05$. SSI: superficial surgical site infection; PE: pulmonary embolism; DVT: deep venous thrombosis; UTI: urinary tract infection

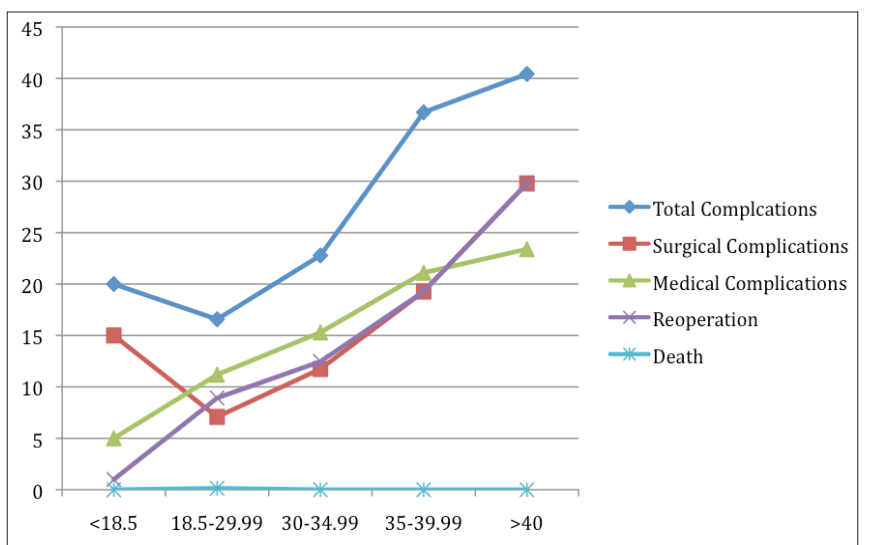

Figure 2: Incidence of adverse events $v s$. body mass index range, for autologous breast reconstruction cohort

while obese patients tended to be older ( 48.0 vs. 51.0 years) [Table 1]. With regards to underweight patients, there was a lower incidence of preoperative commorbidites, with the exception of active smoking $(17 \%$ vs. $13.5 \%)$. With regards to overweight patients, nearly all commorbidities were increased, including hypertension, diabetes, chronic obstructive pulmonary disease (COPD), dyspnea, history of transient ischemic attack (TIA) or cerebrovascular accident (CVA), prior percutaneous coronary intervention (PCI) or previous cardiac surgery (PCS), and chronic steroid use. There was also a significant decrease in outpatient cases, and an increase in work relative value units (RVU) and operative time. These findings are all in accordance with previouslypublished literature. ${ }^{\mid 24,25]}$

Preoperative variables in the autologous group paralleled the prosthetic group [Table 2]. Underweight patients had lower incidence of nearly all comorbidities, with the exception of active smoking, steroid use, and wound infection. Underweight patients had similar percentage of outpatient cases, and decreased operative time. As expected, obese patients had an increased incidence of hypertension, diabetes, dyspnea, and wound infection in the prior 30 days. ${ }^{|24,25|}$ Significantly fewer obese patients were outpatient surgery, and operative time was significantly longer $(6.09 \mathrm{~h}$ vs. $6.61 \mathrm{~h})$.

While it has previously been found that underweight patients tend to utilize prosthetic breast reconstruction to a higher degree, and that obese patients utilize more autologous reconstruction, this is the first national evaluation of this trend. ${ }^{[30]}$ Ostensibly, this phenomenon is the result of the lack of donor-site availability in underweight (as opposed to overweight) patients. However, advanced microsurgical techniques, use of flap plus implant techniques, and doublefree flap techniques have all contributed to increased the availability of autologous reconstruction for underweight patients. ${ }^{[31,32]}$

In our study, we have opted to utilize a unique stratification method, to examine if different BMI categories result in different outcomes. In general, increasing obesity led to statistically increased rates of surgical complications, irrespective of reconstructive type. This is consistent with previous literature on this subject. ${ }^{[3,34]}$ Specifically, wound infection (superficial/deep/organ space), dehiscence, and prosthesis/flap failure all increased as patient BMI 
Table 5: Body mass index as a predictor of outcomes following prosthetic breast reconstruction, multivariate analysis

\begin{tabular}{|c|c|c|c|c|c|c|c|c|c|c|c|c|}
\hline \multirow{3}{*}{$\begin{array}{l}\text { BMI category } \\
<18.5\end{array}$} & \multicolumn{4}{|c|}{ Surgical Complications } & \multicolumn{4}{|c|}{ Medical Complications } & \multicolumn{4}{|c|}{ Reoperation } \\
\hline & \multirow{2}{*}{$\begin{array}{c}\text { OR } \\
0.53\end{array}$} & \multicolumn{2}{|c|}{$95 \% \mathrm{Cl}$} & \multirow{2}{*}{$\begin{array}{c}\boldsymbol{P} \\
0.54\end{array}$} & \multirow{2}{*}{$\begin{array}{c}\text { OR } \\
0.46\end{array}$} & \multicolumn{2}{|c|}{$95 \% \mathrm{Cl}$} & \multirow{2}{*}{$\frac{P}{0.44}$} & \multirow{2}{*}{$\frac{\text { OR }}{0.57}$} & \multicolumn{2}{|c|}{$95 \% \mathrm{Cl}$} & \multirow{2}{*}{$\begin{array}{c}\boldsymbol{P} \\
0.25\end{array}$} \\
\hline & & 0.07 & 3.93 & & & 0.06 & 3.36 & & & 0.26 & 1.33 & \\
\hline 18.5-29.99 & \multicolumn{3}{|c|}{ Reference } & & \multicolumn{3}{|c|}{ Reference } & \multicolumn{5}{|c|}{ Reference } \\
\hline $30-34.99$ & 1.348 & 0.812 & 2.238 & 0.249 & 2.752 & 1.289 & 5.873 & $0.009^{*}$ & 0.983 & 0.585 & 1.653 & 0.949 \\
\hline $35-39.99$ & 2.032 & 1.113 & 3.71 & $0.021^{*}$ & 2.13 & 0.746 & 6.082 & 0.158 & 2.018 & 1.154 & 3.528 & $0.014^{*}$ \\
\hline$>40$ & 3.308 & 1.709 & 6.403 & $<0.001^{*}$ & 0.591 & 0.075 & 4.654 & 0.617 & 1.893 & 0.914 & 3.921 & 0.086 \\
\hline
\end{tabular}

*Denotes significant value, $P<0.05$. BMI: body mass index; OR: odds ratio; $\mathrm{Cl}$ : confidence interval

Table 6: Body mass index as a predictor of outcomes following autologous breast reconstruction, multivariate analysis

\begin{tabular}{|c|c|c|c|c|c|c|c|c|c|c|c|c|}
\hline \multirow{3}{*}{$\begin{array}{l}\begin{array}{l}\text { BMI } \\
\text { category }\end{array} \\
<18.5\end{array}$} & \multicolumn{4}{|c|}{ Surgical Complications } & \multicolumn{4}{|c|}{ Medical Complications } & \multicolumn{4}{|c|}{ Reoperation } \\
\hline & \multirow{2}{*}{$\frac{\mathrm{OR}}{2.48}$} & \multicolumn{2}{|c|}{$95 \% \mathrm{Cl}$} & \multirow{2}{*}{$\begin{array}{c}\boldsymbol{P} \\
0.07\end{array}$} & \multirow{2}{*}{$\frac{\text { OR }}{0.66}$} & \multicolumn{2}{|c|}{$95 \% \mathrm{Cl}$} & \multirow{2}{*}{$\frac{\boldsymbol{P}}{0.44}$} & \multirow{2}{*}{$\begin{array}{c}\text { OR } \\
0.72\end{array}$} & \multicolumn{2}{|c|}{$95 \% \mathrm{Cl}$} & \multirow{2}{*}{$\begin{array}{c}\boldsymbol{P} \\
0.68\end{array}$} \\
\hline & & 0.85 & 6.88 & & & 0.23 & 2.33 & & & 0.17 & 3.14 & \\
\hline 18.5-29.99 & \multicolumn{3}{|c|}{ Reference } & & \multicolumn{3}{|c|}{ Reference } & \multicolumn{5}{|c|}{ Reference } \\
\hline $30-34.99$ & 1.808 & 1.127 & 2.9 & $0.014^{*}$ & 1.203 & 0.787 & 1.839 & 0.394 & 1.319 & 0.835 & 2.082 & 0.235 \\
\hline $35-39.99$ & 3.357 & 1.902 & 5.925 & $<0.001^{*}$ & 1.699 & 0.974 & 2.964 & 0.062 & 2.237 & 1.269 & 3.943 & $0.005^{*}$ \\
\hline$>40$ & 5.552 & 2.748 & 11.218 & $<0.001^{*}$ & 1.857 & 0.868 & 3.97 & 0.111 & 4.144 & 2.038 & 8.427 & $<0.001^{*}$ \\
\hline
\end{tabular}

*Denotes significant value, $P<0.05$. BMI: body mass index; OR: odds ratio; $\mathrm{Cl}$ : confidence interval

increased, although differences were more exaggerated in the autologous reconstruction group [Tables 3 and 4]. This finding was confirmed on both univariate and multivariate analysis.

Multiple medical complications increased as patient weight increased, in both reconstructive groups. Specifically, bleeding requiring transfusion and DVT were elevated in the prosthetic group; and PE, acute renal failure, and sepsis/ septic shock were elevated in the autologous group. On multivariate analysis, while medical complication rates were elevated, data only reached significance for the prosthetic in moderate obesity group (OR $2.752, P=0.009)$. Finally, reoperation rates were significantly elevated in both stratified obesity cohorts, with a stronger relationship in the autologous reconstruction group.

Previous literature has suggested that underweight patients suffer from elevated rates of surgical complications, and specific catastrophic medical complications (including death). While we found elevated rates of surgical complications in the autologous reconstruction group and medical complications in the prosthetic reconstruction group, we otherwise found a decreased incidence of surgical and medical complications, reoperation and death in underweight patients. However, none of these findings were significant on multivariate analysis. These findings suggest that, as with previous studies, patient groups may be too small to yield significant differences. Given the relatively small size of underweight breast reconstruction patients captured in NSQIP, it is not possible to discern between patients with lean muscle mass, versus those with chronic disease and multiple comorbidities. As the dataset continues to grow, it will be possible to separate these groups, thus increasing the value of data extracted from the dataset. However, at this time, our findings suggest that all forms of breast reconstruction are safe in underweight patients. Additionally, there does not appear to be a role for the "obesity paradox" in breast reconstruction.

In conclusion, this study represents the only review to date of post-mastectomy breast reconstruction, using a weightstratification system. Increasing obesity is associated with significantly increased risk of adverse events (AE's) in the first 30 days following breast reconstruction. The added risks translate into higher rates of overall morbidity, regardless of reconstructive modality. On multivariable analysis of over 4,600 patients, there were no significant differences in the rates of adverse events between underweight patients (BMI $<18.5$ ) and their reference-weight counterparts, in spite of a significant increase in surgical and medical complication rates in underweight patients on univariate analysis. Based on the overall analysis, we conclude that while obese patients are at greater risk when undergoing breast reconstruction, with appropriate counseling breast reconstruction should continue to be offered to these patients.

\section{Financial support and sponsorship Nil.}

\section{Conficts of interest}

There are no conflicts of interest.

\section{REFERENCES}

I. World Health Organization. Fact sheet No 31।: Obesity and overweight. Geneva:World Health Organization. 2012.

2. Ogden $\mathrm{CL}$, Carroll MD, Kit BK, Flegal KM. Prevalence of obesity in the United States, 2009-2010. NCHS Data Brief 20I2;(82): I-8.

3. Russell GV, Pierce CW, Nunley L. Financial implications of obesity. Orthop Clin North Am 201 I;42:123-7.

4. Arterburn, DE, Maciejewski ML Tsevat J. Impact of morbid obesity on medical expenditures in adults. Int J Obes (Lond) 2005;29:334-9. 
5. Abedi NN, Davenport DL, Xenos E, Sorial E, Minion DJ, Endean ED. Gender and 30-day outcome in patients undergoing endovascular aneurysm repair (EVAR): an analysis using the ACS NSQIP dataset. J Vasc Surg 2009 Sep;50:486-9I.

6. Mullen JT, Davenport DL, Hutter MM, Hosokawa PW, Henderson WG, Khur SF, Moorman DW. Impact of body mass index on perioperative outcomes in patients undergoing major intra-abdominal cancer surgery. Ann Surg Oncol 2008;15:2164-72.

7. Mullen JT, Moorman DW, Davenport DL. The obesity paradox: body mass index and outcomes in patients undergoing nonbariatric general surgery. Ann Surg 2009;250:166-72.

8. Tremblay A, Bandi V. Impact of body mass index on outcomes following critical care. Chest 2003; I23:1202-7.

9. O’Brien JM Jr, Phillips GS, Ali NA, Lucarelli M, Marsh CB, Lemeshow S. Body mass index is independently associated with hospital mortality in mechanically ventilated adults with acute lung injury. Crit Care Med 2006;34:738-44

10. Curtis JP, Selter JG, Wang Y, Rathore SS, Jovin IS, Jadbabaie F, Kosiborod M, Portnay EL, Sokol SI, Bader F, Krumholz HM.. The obesity paradox: body mass index and outcomes in patients with heart failure. Arch Intern Med 2005;|65:55-6I.

II. Galanos AN, Pieper CF, Kussin PS, Winchell MT, Fulkerson WJ, Harrell FE Jr Teno JM, Layde P, Connors AF Jr, Phillips RS, Wenger NS. Relationship of body mass index to subsequent mortality among seriously ill hospitalized patients. SUPPORT Investigators. The Study to Understand Prognoses and Preferences for Outcome and Risks of Treatments. Crit Care Med 1997;25:1962-8

12. Fleischmann E, Teal N, Dudley J, May W, Bower JD, Salahudeen AK.. Influence of excess weight on mortality and hospital stay in 1346 hemodialysis patients. Kidney Int 1999;55:I560-7.

13. Fonarow GC, Srikanthan P, Costanzo MR, Cintron GB, Lopatin M; ADHERE Scientific Advisory Committee and Investigators. An obesity paradox in acute heart failure: analysis of body mass index and inhospital mortality for 108,927 patients in the Acute Decompensated Heart Failure National Registry. Am Heart J 2007; I53:74-8I

14. Gruberg L,Weissman NJ,Waksman R, Fuchs S, Deible R, Pinnow EE, Ahmed LM, Kent KM, Pichard AD, Suddath WO,Satler LF, Lindsay J Jr. The impact of obesity on the short-term and long-term outcomes after percutaneous coronary intervention: the obesity paradox? J Am Coll Cardiol 2002;39:57884.

15. Gurm HS, Brennan DM, Booth J, Tcheng JE, Lincoff AM, Topol EJ. Impact of body mass index on outcome after percutaneous coronary intervention (the obesity paradox). Am J Cardiol 2002;90:42-5.

16. Christian CK, Niland J, Edge SB, Ottesen RA, Hughes ME, Theriault R, Wilson J, Hergrueter CA, Weeks JC. A multi-institutional analysis of the socioeconomic determinants of breast reconstruction: a study of the National Comprehensive Cancer Network. Ann Surg 2006;243:24I-9.

17. Alderman AK, Wilkins EG, Kim HM, Lowery JC. Complications in postmastectomy breast reconstruction: two-year results of the Michigan Breast Reconstruction Outcome Study. Plast Reconstr Surg 2002; 109:226574
18. Atisha DM, Alderman AK, Kuhn LE, Wilkins EG. The impact of obesity on patient satisfaction with breast reconstruction. Plast Reconstr Surg 2008; | $21: 1893-9$.

19. Chapman GW Jr, Mailhes JB, Thompson HE. Morbidity in obese and nonobese patients following gynecologic surgery for cancer.J Natl Med Assoc 1988;80:417-20.

20. Foley K, Lee RB. Surgical complications of obese patients with endometria carcinoma. Gynecol Oncol 1990;39: I71-4.

21. Jiganti JJ, Goldstein WM, Williams CS. A comparison of the perioperative morbidity in total joint arthroplasty in the obese and nonobese patient. Clin Orthop Relat Res 1993:175-9.

22. Stern SH, Insall JN. Total knee arthroplasty in obese patients.J Bone Joint Surg Am. 1990;72:1400-4.

23. Vinton AL, Traverso LW, Jolly PC. Wound complications after modified radical mastectomy compared with tylectomy with axillary lymph node dissection. Am / Surg 1991;161:584-8.

24. Thomas EJ, Goldman L, Mangione CM, Marcantonio ER, Cook EF, Ludwig L, Sugarbaker D, Poss R, Donaldson M, Lee TH.. Body mass index as a correlate of postoperative complications and resource utilization. Am J Med 1997; 102:277-83.

25. Klasen J, Junger A, Hartmann B, et al. Increased body mass index and peri-operative risk in patients undergoing non-cardiac surgery. Obes Surg 2004;|4:275-8|.

26. Dindo D, Muller MK, Weber M, Clavien PA. Obesity in general elective surgery. Lancet 2003;36I:2032-5.

27. Kim EK, Eom JS, Hwang $\mathrm{CH}$, Ahn SH, Son BH, Lee TJ. Immediate transverse rectus abdominis musculocutaneous (TRAM) flap breast reconstruction in underweight Asian patients. Breast Cancer 20I4;21:693-7.

28. Kroll SS. Bilateral breast reconstruction in very thin patients with extended free TRAM flaps. Br J Plast Surg 1998;51:535-7.

29. Lee JH, Lee PK, Oh DY, Rhie JW, Ahn ST. Subpectoral-subfascial breast augmentation for thin-skinned patients. Aesthetic Plast Surg 2012;36:I I5-2I.

30. Kronowitz SJ, Robb GL, Youssef A, Reece G, Chang SH, Koutz CA, Ng RL, Lipa JE, Miller MJ. Optimizing autologous breast reconstruction in thin patients. Plast Reconstr Surg 2003; I I 2: 1768-78.

3I. Figus A, Fioamonti P, Ramakrishnan V. Stacked free SIEA/DIEP flap for unilateral breast reconstruction in a thin patient with an abdominal vertical midline scar.J Reconstr Microsurg 2007;23:523-5.

32. Kitcat M, Molina A, Meldon C, Darhouse N, Clibbon J, Malata CM. A Simple Algorithm for Immediate Postmastectomy Reconstruction of the Smal Breast-A Single Surgeon's 10-Year Experience. Eplasty 2012; I 2:e55.

33. Albornoz CR, Cordiero PG, Farias-Eisner G, Mehrara BJ, Pusic AL, McCarthy CM, Disa JJ, Hudis CA, Matros E. Diminishing relative contraindications for immediate breast reconstruction. Plast Reconstr Surg 20I4;134:e363-9.

34. Nguyen KT, Hanwright PJ, Smetona JT, Hirsch EM, Seth AK, Kim JY. Body mass index as a continuous predictor of outcomes after expander-implant breast reconstruction. Ann Plast Surg 2014;73:19-24 